\title{
Pars Plana Vitrectomy for Symptomatic Vitreous Floaters: Another Look
}

\author{
Tayab C Waseem, PhD, Evan R DaBreo, MD, Jiang Douglas, MS, Yousef Hasanzadah, \\ BS, Rebecca Clawson, BS, Alan L Wagner, MD, FACS and Kapil G Kapoor, MD, FACS*
}

Wagner Macula \& Retina Center, Eastern Virginia Medical School, USA

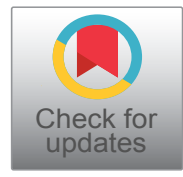

*Corresponding author: Kapil G. Kapoor, MD, FACS, Wagner Macula \& Retina Center, 5520 Greenwich Road Suite 204, Virginia Beach, VA 23462, USA, Tel: 757-481-4400, Fax: 757-481-1285

\begin{abstract}
Introduction: Historically, pars plana vitrectomy (PPV) has been considered a controversial treatment for elective removal of primary symptomatic vitreous opacities (floaters) due to the possibility of extreme and even blinding side effects of the procedure. The purpose of this study is to determine the safety and patient satisfaction level for those who undergo PPV for removal of vitreous floaters.
\end{abstract}

Methods: This was a retrospective study of 54 eyes in 51 patients (average age 68) who underwent 23 gauges PPV between 2014 and 2017. We assessed the rate of intraoperative and postoperative complications at a minimum 1-month follow-up (average 12.63 months, range 1.0337.78).

Results: There was a lack of sight-threatening complications in $100 \%$ of patients $(n=51) .100 \%$ of patients surveyed noted subjective improvement following floaterectomy. Three patients $(5.6 \%)$ developed epiretinal membrane, one patient developed posterior uveitis $(1.8 \%)$, and one patient developed a new vitreous hemorrhage (1.8\%). One patient also developed a possible bulbar neuritis (1.8\%). There were no cases of endophthalmitis, cataracts, or retinal tears.

Conclusion: PPV can be a safe and efficacious surgical therapy to improve patient quality of life by improving symptoms from floaters.

\section{Keywords}

Vitreous floaters, Vitreolysis, Retina, Floaterectomy

\section{Introduction}

Primary symptomatic vitreous opacities, often referred to as 'floaters' are a common presenting com- plaint which until recently was not often considered for surgical intervention [1-3]. Primary vitreous floaters are the results of aggregated endogenous collagen fibrils of the vitreous body which disrupt and scatter light on its path to the retina. Vitreous floaters can be progressive in young patients with axial myopia and older patients due to aging as the vitreous body liquefies and bundled collagen fibrils become thickened and more numerous $[3,4]$. Primary vitreous floaters of sudden onset can be the result of posterior vitreous detachment often resulting in a Weiss Ring. Secondary vitreous floaters are those with an etiology which is not endogenous to the vitreous body, most often due to vitreous hemorrhage, or due to the accumulation of other exogenous material (iatrogenic medication, inflammatory debris, retinal pigmented cells in RD) [3]. For most patients, floaters are of little consequence due to adaptation or natural resolution [1-3]. However, for a minority of patient's floaters are a debilitating pathology resulting in a reduction of quality of life. Because objective measures, such as visual acuity, are often unaffected by floaters most treatment is driven by patient desire for improvement of symptoms [5-9].

More recently, vitreolysis or photo dissolution of vitreous floaters using $\mathrm{Nd}$ : YAG laser has gained increasing popularity. Its recent prominence is interesting given its lack of novelty and scarcity of prospective clinical trial data supporting its use. One recent randomized trial did evaluate YAG vitreolysis vs. sham laser for symptomatic Weiss ring floaters from posterior vitreous detachment only and at 6 months found a $53 \%$ subjective improve-

Citation: Waseem TC, DaBreo ER, Douglas J, Hasanzadah Y, Clawson R, et al. (2021) Pars Plana Vitrectomy for Symptomatic Vitreous Floaters: Another Look. Int J Ophthalmol Clin Res 8:124. doi. org/10.23937/2378-346X/1410124

Accepted: March 20, 2021: Published: March 22, 2021

Copyright: (C) 2021 Waseem TC, et al. This is an open-access article distributed under the terms of the Creative Commons Attribution License, which permits unrestricted use, distribution, and reproduction in any medium, provided the original author and source are credited. 
ment rate. While this marks an improvement from a previous study by Delaney, et al. describing $38 \%$ of patients subjectively improving from vitreolysis, it is still underwhelming [3]. Further, some patients had challenges in the trial - with one patient having lens pitting and an additional patient having worsening symptomatic floaters after vitreolysis that ultimately required pars plana vitrectomy [10]. There have additionally been several reports of complications associated with YAG vitreolysis outside of the boundaries of this clinical trial, as outlined recently by a report from the American Society of Retina Specialists Research and Safety in Therapeutics (ASRS ReST) Committee [11].

Increasing concerns regarding these safety reports and the underwhelming subjective improvements of vitreolysis encourages us to reevaluate the spectrum of treatment for symptomatic vitreous floaters. There have been some reports on PPV for floaters in the past with moderate success. Both Schiff, et al. and Sebag have demonstrated excellent efficacies as one might expect with excellent subjective improvement, but there were some noted complications that were sight-threatening in a small percentage of patients. In an effort to be less invasive, a recent report on partial vitrectomy demonstrated similarly excellent success rates but still had a notable level of rhegmatogenous complications (1.5\%).

Given the increasing use of sutureless small gauge vitrectomy, an updated appraisal of the risks and subjective improvement of vitrectomy for vitreous floaters is critical. This study aims to evaluate the clinical outcomes of our experience with pars plana vitrectomy (PPV) for vitreous floaters.

\section{Methods}

Clinical records were retrospectively reviewed for 54 eyes of 51 patients who underwent PPV for symptomatic vitreous floaters at our centers. All patients enrolled in the review had a history of $\geq 6$ months' of symptomatic vitreous opacities which were debilitating enough for the patient to seek surgical options through a referral. All patients had a comprehensive ophthalmologic examination and an extensive discussion of risks, benefits and alternatives prior to proceeding with PPV for symptomatic vitreous floaters.

PPV can be used to treat many conditions other than vitreous floaters, such as epiretinal membrane, vitreous hemorrhage, macular pucker, and retinal detachment. Patients who underwent PPV primarily for these conditions were excluded. Patients with less than 1 month of follow up time were also excluded from this study. The PPV's were carried out under monitored anesthesia care (MAC) using 23 gauge needles by two ophthalmic surgeons in two hospitals within the Sentara and Bon Secours medical network. Pre-operative visits and follow up visits were carried out in one of nine outpa- tient clinics. PPV was followed by a course of Maxitrol ointment four times daily for one week, then tapered to three times daily for 3 days, two times daily for 3 days, 1 time daily for 3 days, then discontinued.

Follow-up was done post-operatively on an average schedule of 1 day, 10 days, 6 weeks, and 3 months, which included the above-mentioned ophthalmic evaluations. As per our standard clinical practice at least one of these post-operative visits was scheduled with a retinal surgeon in the practice that was not the primary surgeon. Part of the post-operative evaluation included the primary surgeon evaluating an improvement in vitreous floaters from the pre-operative state and the non-operative surgeon confirming the objective absence of any residual vitreous opacities that could be visually significant. Adverse post-operative outcomes were recorded to include the occurrence of sight-threatening complications (asymptomatic retinal tears, symptomatic retinal tears, retinal detachment, cataract formation and endophthalmitis).

Best corrected visual acuity (BCVA) values were taken with Snellen eye chart examinations. The BCVA values were taken immediately at pre-op and at each follow up visit. For comparison, BCVA values were recorded from immediately pre-op and at the 3-month follow up visit. These values were converted to the logMAR scale using the equation "LogMAR VA = LogMAR value of the best line read $-0.02 \times$ (number of letters read)". The LogMAR value of best line read can be retrieved from Table 1. The mean and standard deviation of the logMAR values taken pre-operatively and 3 months post-operatively were then taken and compared.

\section{Results}

A total of 54 eyes in 51 patients underwent PPV for the treatment of symptomatic vitreous opaci-

Table 1: Conversion of Snellen eye chart examination score (BCVA score) converted to LogMAR scale using the equation LogMAR VA = LogMAR value of the best line read $-0.02 X$ (number of letters read).

\begin{tabular}{|l|l|}
\hline \multicolumn{2}{|l|}{ Snellen Visual Acuity to LogMAR Conversion } \\
\hline Snellen & LogMAR \\
\hline & 0 \\
\hline $20 / 20$ & +0.1 \\
\hline $20 / 25$ & +0.2 \\
\hline $20 / 32$ & +0.3 \\
\hline $20 / 40$ & +0.4 \\
\hline $20 / 50$ & +0.5 \\
\hline $20 / 63$ & +0.6 \\
\hline $20 / 80$ & +0.7 \\
\hline $20 / 100$ & +0.8 \\
\hline $20 / 125$ & +0.9 \\
\hline $20 / 160$ & +1.0 \\
\hline $20 / 200$ & \\
\hline
\end{tabular}


ties. Table 2 displays the characteristics of the eyes followed. 22 eyes were OS (41\%) and 32 eyes were on the OD $(60 \%)$. The mean and median age of patients was 66 (range 45-87). The mean follow-up was 16.8 months (range, 3.2-33.8). 24 patients were male $(44 \%)$ and 30 patients were female (55\%). $85.2 \%$ ( $n$ $=46$ ) of patients had a diagnosis of primary vitreous floaters due to posterior vitreous detachment. Other causes of primary vitreous floaters include vitreous synchysis $(5.6 \%)$, asteroid hyalosis $(5.6 \%)$, vitreous hemorrhage $(1.9 \%)$, and panuveitis secondary to autoimmune disease (1.9\%).

At the time of the surgery, 42 eyes were pseudophakic (78\%) and 42 eyes had PVD (78\%). 11 eyes had nucle-

Table 2: Characteristics of each patient and each eye that underwent pars plana vitrectomy for removal of vitreous floaters.

\begin{tabular}{|l|l|}
\hline Number of patients & 54 \\
\hline Number of Eyes & 51 \\
\hline Gender & \\
\hline Male & $24(44.4 \%)$ \\
\hline Female & $30(55.6 \%)$ \\
\hline Age & \\
\hline Mean (range) & $68.1(49-87)$ \\
\hline Follow up (months) & \\
\hline Mean (range) & $12.63(1.03-37.78)$ \\
\hline Median & 10.1 \\
\hline Eyes & \\
\hline OS & $22(41 \%)$ \\
\hline OD & $32(60 \%)$ \\
\hline Vitrectomy & \\
\hline 23 Gauge under MAC & $54(100 \%)$ \\
\hline Existing pathology in effected eye & \\
\hline Pseudophakia & $42(77.8 \%)$ \\
\hline PVD & $46(85.2 \%)$ \\
\hline Nuclear sclerosis & $11(20 \%)$ \\
\hline Glaucoma & $5(9.3 \%)$ \\
\hline Lattice degeneration & $4(7.4 \%)$ \\
\hline Cystoid macular edema & $3(5.6 \%)$ \\
\hline Diabetic retinopathy & $2(3.7 \%)$ \\
\hline Retinal detachment without tear & $2(3.7 \%)$ \\
\hline Macular hole & $2(3.7 \%)$ \\
\hline & \\
\hline
\end{tabular}

ar sclerosis (20\%) and 5 eyes had glaucoma (9\%). Several other pathologies present at the time of surgery are presented.

In the minimum follow-up period of 1 month, there were no sight threatening complications, as defined above, in $100 \%$ of patients $(n=51) .11 .1 \%(n=6)$ of patients had new pathologies arise during the course of follow-up. Table 3 presents all post-operative complications. Three patients (5.6\%) developed epiretinal membrane, one patient developed posterior uveitis (1.8\%), and one patient developed a new vitreous hemorrhage (1.8\%). One patient also developed a possible bulbar neuritis (1.8\%). There were no cases of endophthalmitis, cataracts, or retinal tears.

Epiretinal membrane occurred in 3 eyes (5.6\%) at 3 weeks, 3 months, and 5.5 months after surgery. Epiretinal membrane occurred 3 weeks after surgery in a 58-year-old male with vitreous debris secondary to PVD and vitreomacular traction syndrome. Observation was recommended. A recurrent and worsening epiretinal membrane was reported in a 77-year-old female with pseudophakia and PVD 3 months after surgery. Observation was recommended, and the patient was given the option for further surgery if needed. A symptomatic Epiretinal membrane with a pseudohole significantly effecting ADL developed in a pseudophakic 70-year-old male with PVD 5.5 months after surgery. A 23g PPV under MAC with ILM stripping was carried out with resolution of symptoms. Posterior uveitis developed in 1 eye (1.8\%) 1 month after surgery in a 73-year-old female with pseudophakia and PVD who was placed under observation after having ESR/CRP labs done. Vitreous hemorrhage developed at 1 month after surgery in 1 eye $(1.8 \%)$ belonging to a 75 -year-old male which spontaneously cleared within a month of onset. 1 eye (1.8\%) in a 65-year-old male presented 9 months after surgery with eye pain thought to be a possible bulbar neuritis which was referred to a neurologist for assessment.

Visual acuities did not significantly change after PPV (Figure 1). 46 (85.2\%) of eyes had more than 3 months of follow up time and were included in this comparison. Preoperative visual acuity converted to LogMAR was 0.31 with a standard deviation of 0.28 while the post-operative VA was 0.28 with a standard deviation of 0.27 . A paired $t$-test revealed no significant difference between pre-op and post-op logMAR visual acuities $(P=$

Table 3: Post-operative complications observed in patients following pars plana vitrectomy and course of action taken.

\begin{tabular}{|l|l|l|l|}
\hline Complication & Percent & Time of Onset & Course of Action \\
\hline Epiretinal Membrane & $5.6 \%(n=3)$ & $\begin{array}{l}3 \mathrm{wk} \\
3 \mathrm{mo} \\
5.5 \mathrm{mo}\end{array}$ & $\begin{array}{l}\text { Observation } \\
\text { Observation } \\
\text { PPV with ILM stripping }\end{array}$ \\
\hline Posterior Uveitis & $1.8 \%(\mathrm{n}=1)$ & mo & Observation and labs \\
\hline Vitreous hemorrhage & $1.8 \%(n=1)$ & $1 \mathrm{wk}$ & Cleared spontaneously after 1 month \\
\hline Possible bulbar neuritis & $1.8 \%(n=1)$ & $9 \mathrm{mo}$ & Referred to neurology \\
\hline
\end{tabular}


Pre-operative LogMAR VA $\square$ Post-operative LogMAR VA

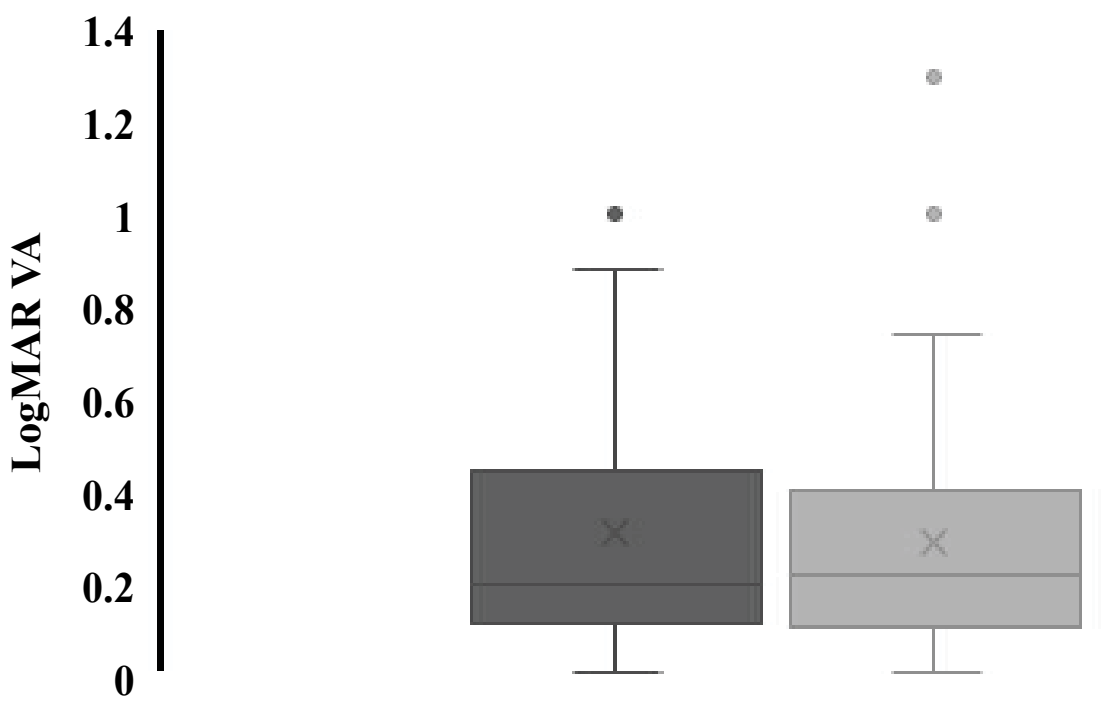

Figure 1: Pre-operative VA compared to post-operative VA following pars plana vitrectomy.

0.24). 14 eyes also utilized a pinhole exam during examination. Within the pinhole visual acuities, the logMAR VA pre-operatively was 0.27 (SD 0.17) and the VA mean post-operatively was 0.26 (SD 0.16). No significant difference was found ( $p=0.43)$.

There was objective improvement in vitreous floaters affecting ADL in $100 \%(n=54)$ of patients as observed by the primary surgeon and non-operative surgeon. In the immediate post-operative period all patients were surveyed on their improvement based on relief of symptoms from floaters and there was a $100 \%$ subjective improvement rate. Subsequently, between 1 and 3 years after the procedure, patients were surveyed by an independent observer regarding their improvement of symptoms after vitrectomy and $100 \%$ of those that were reached had persistent subjective improvement. Despite a large variation in follow-up time (mean 16.8 months, range, 3.2-33.8 month), no statistical significance was found regarding the relationship between overall satisfaction and short vs. long follow-up (Chisquare analysis; $p>0.05$ ).

\section{Discussion and Conclusion}

Primary vitreous floaters have been a difficult complaint to manage as the degree of distress experienced by some patient is not well reflected by common metrics such as visual acuity $[1,3,12]$. While most patients express very little distress over their floaters, a small minority of patients find their floaters extremely bothersome, reporting impairment in daily activities such as driving, reading small text and employments which requires fine detail work $[1,3]$. Due to the prevalence of floaters, and their frequent occurrence as a result of natural aging, many view floaters as not clinically significant and therefore not necessary to treat $[3,5,7,13]$.
However, given the largely subjective nature of floater complaints it is important to look at patient centered outcomes such as patient satisfaction and reported improvement in activities of daily living $[1,13]$. Numerous recent papers have shown high levels of patient satisfaction with PPV for SVO [14,15].

Previous studies on the efficacy of a full vitrectomy have shown an extensive rate of cataract formation after surgery, however cataract surgery was not indicated for any of the eyes followed in this study. A study by Sebag, et al. demonstrated the efficacy of a limited vitrectomy vs. full vitrectomy by showing a lower rate of cataract formation [16]. However, the evidence in our study suggests that cataract formation is not necessarily higher following full vitrectomies than after limited vitrectomies.

As seen in our study and others, PPV can be an efficacious first option for symptomatic vitreous floaters. There were important limitations of our study. First, this was a retrospective study and future prospective randomized trials are needed to fully evaluate PPV vs. vitreolysis. Second, subjective data from patients was tabulated as binary data (yes/no) and qualifying this further on a scale would lend a greater picture to the extent of their improvement. Also, this was a single center study involving two surgeons, and expanding the series to more centers may lend a greater representation of real-world vitrectomy data.

Currently, there is no clear consensus among ophthalmologists when it is appropriate to treat symptomatic vitreous floaters with PPV $[17,18]$. Currently available data suggests that PPV provides better visual outcomes for patients with floaters compared with YAG vitreolysis, although there has been limited re- 
ports comparing these two modalities [17,19]. As more reports of outcomes following vitrectomy for vitreous floaters become available, the field can more quickly arrive at a consensus that is supported by the most up to date evidence.

\section{References}

1. Ivanova T, Jalil A, Antoniou $\mathrm{Y}$, Bishop PN, Vallejo-Garcia $\mathrm{JL}$, et al. (2016) Vitrectomy for primary symptomatic vitreous opacities: An evidence-based review. Eye 30: 645-655.

2. Milston R, Madigan MC, Sebag J (2016) Vitreous floaters: Etiology, diagnostics, and management. Surv Ophthalmol 61: 211-227.

3. Delaney YM, Oyinloye A, Benjamin L (2002) Nd: YAG vitreolysis and pars plana vitrectomy: surgical treatment for vitreous floaters. Eye 16: 21-26.

4. Bos K, Holmes D, Meadows R, Kadler K, Mcleod D, et al. (2001) Collagen fibril organisation in mammalian vitreous by freeze etch/rotary shadowing electron microscopy. Micron 32: 301-306.

5. Sebag J (2014) Reassessing the surgical treatment of floaters. Retina Today.

6. Zou H, Liu H, Xu X, Zhang X (2012) The impact of persistent visually disabling vitreous floaters on health status utility values. Qual Life Res 22: 1507-1514.

7. Wagle AM, Lim WY, Yap TP, Neelam K, Au Eong KG (2011) Utility values associated with vitreous floaters. Am J Ophthalmol 152: 60-65.

8. Mossa F, Delaney YM, Rosen PH, Rahman R (2002) Floaterectomy: Combined phacoemulsification and deep anterior vitrectomy. J Cataract Refract Surg 28: 589-592.

9. Yonemoto J, Ideta H, Sasaki K, Hirose A, Oka C (1994) The age of onset of posterior vitreous detachment. Graefes Arch Clin Exp Ophthalmol 232: 67-70.
10. Shah CP, Heier JS (2017) YAG Laser Vitreolysis vs. Sham YAG Vitreolysis for Symptomatic Vitreous Floaters: A Randomized Clinical Trial. JAMA Ophthalmol 135: 918-923.

11. Hahn P, Schneider EW, Tabandeh H, Wong RW, Emerson GG (2017) Reported complications following laser vitreolysis. JAMA Ophthalmology 135: 973-976.

12. Tan HS, Mura M, Oberstein SYL, Bijl HM (2011) Safety of vitrectomy for floaters. Am J Ophthalmol 151: 995-998.

13. Sebag J, Yee KM, Wa CA, Huang LC, Sadun AA (2014) Vitrectomy for floaters: Prospective Efficacy Analyses and Retrospective Safety Profile. Retina 34: 1062.

14. Martínez-Sanz F, Velarde JI, Casuso P, Fernández-Cotero JN (2009) Surgical solution to vitreous floaters visual problem. Arch Soc Esp Oftalmol 84: 259-262.

15. de Nie KF, Crama N, Tilanus MA, Klevering BJ, Boon CJ (2013) Pars plana vitrectomy for disturbing primary vitreous floaters: clinical outcome and patient satisfaction. Graefes Arch Clin Exp Ophthalmol 251: 1373-1382.

16. Sebag J, Yee KMP, Nguyen JH, Nguyen-Cuu J (2018) Long-Term Safety and Efficacy of Limited Vitrectomy for Vision Degrading Vitreopathy Resulting from Vitreous Floaters. Ophthalmol Retina 2: 881-887.

17. Broadhead GK, Hong T, Chang AA (2020) To Treat or Not to Treat: Management Options for Symptomatic Vitreous Floaters. Asia Pac J Ophthalmol (Phila) 9: 96-103.

18. Henry CR, Smiddy WE, Flynn HW Jr. (2014) Pars plana vitrectomy for vitreous floaters: Is there such a thing as minimally invasive vitreoretinal surgery? Retina 34: 1043-1045.

19. Kokavec J, Wu Z, Sherwin JC, Ang AJS, Ang GS (2017) Nd: YAG laser vitreolysis versus pars plana vitrectomy for vitreous floaters. Cochrane Database Syst Rev 6: CD011676. 